\title{
Functionalized carbon nanotubes: revolution in brain delivery
}

\author{
"...there are increasing numbers of studies documented on the use of \\ functionalized carbon nanotubes for brain delivery."
}

Keywords: blood-brain barrier • brain drug delivery $\bullet$ carbon nanotubes

\begin{abstract}
Biocompatible functionalized carbon nanotubes

Carbon nanotubes (CNTs) are firstly described by Iijima in early nineties as needle-like tubes composed of layers of graphitic sheets [1]. Either single-walled or multiwalled carbon nanotubes (SWNTs or MWNTs), made of one or several layers of graphene, have been utilized mainly in industry due to their characteristic high mechanical strength, good electronic and thermal properties [2]. Together with the large surface area, the relatively low weight makes them ideal particularly for applications in neuroengineering [3]. However, due to the hydrophobic nature, pristine CNTs cannot be functionally integrated into biological systems unless they undergo surface fictionalization to become more biocompatible. Many approaches have been developed to alter the inert property of CNTs either through covalent (e.g., oxidation and chemical conjugation) or noncovalent (e.g., coating with hydrophilic or biological molecules) strategies [4]. Since then, functionalized CNTs ( $f$-CNTs), possessing reduced cytotoxicity, have received tremendous attention as nanomaterials in biomedicine. In particular, $f$-CNTs held great promise as drug or gene carriers in vitro and in vivo $[5,6]$.
\end{abstract}

\section{Studies of carbon nanotubes in brain for therapeutic applications}

The journey for $f$-CNTs, serving as drug- and gene-delivery vectors, starts with their cellular internalization. Entering cells through macropinocytosis was proposed by Bhattacharta et al. as accumulation of SWNT-
DNA hybrids in vacuoles was observed in human umbilical vein endothelial cells by transmission electron microscopy (TEM) [7]. It has also been found that $f$-CNTs can directly penetrate cell membranes in an energy-independent manner possibly due to their 'needle-like' structure $[8,9]$. Delivering drugs into brain is rather challenging and is greatly limited due to the presence of the blood-brain barrier (BBB) [10]. Nevertheless, many studies have demonstrated the success of using $f$-CNTs for brain delivery. Some of the important studies are discussed in the following paragraphs.

Two recently published studies have documented the capability of $f$-CNTs to cross the BBB using in vitro models. Shityakov et al. assessed the dynamic transport of FITC-labeled MWNTs across immortalized murine microvascular cEND cells using the Transwell ${ }^{\circledR}$ device (Corning Inc., NY, USA) [11]. The accumulation of FITCMWNTs in cells over time was imaged by fluorescence and phase-contrast microscopy. The transcellular distribution of FITCMWNTs was 3D reconstructed using dynamics simulation modeling software. FITC-MWNT aggregates were nonfluorescent and localized on the cell surface while more dispersed FITC-MWNTs were located intracellularly or associated with cell membrane. Although fluorescence microscopy could provide information on the temporal and spatial cellular distribution of fluorescently labeled $f$-CNTs, fluorescence was likely to be quenched when $f$-CNTs formed agglomerates. TEM is therefore considered to be the gold standard method to examine the

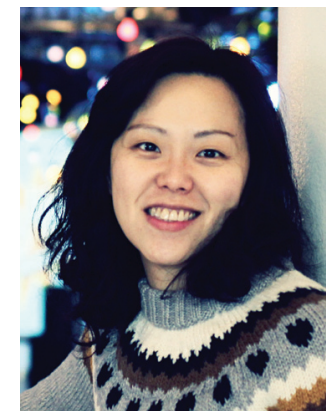

Julie Tzu-Wen Wang Institute of Pharmaceutical Science, Faculty of Life Sciences \& Medicine, King's College London, London, SE1 9NH, UK

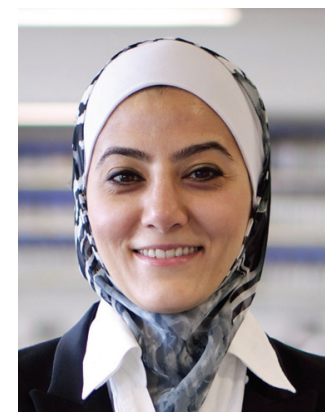

Khuloud Taysir Al-Jamal Author for correspondence: Institute of Pharmaceutical Science, Faculty of Life Sciences \& Medicine, King's College London, London, SE1 9NH, UK

al-jamal@kcl.ac.uk 
interaction of $f$-CNTs with cells by visualizing $f$-CNTs at an ultrastructural level. Another study, conducted in our laboratory, investigated the penetration properties of amino-functionalized MWNTs (MWNT$\mathrm{NH}_{3}^{+}$) using a co-culture Transwell in vitro BBB model comprising of primary porcine brain endothelial cells and primary rat astrocytes [12]. The primary porcine brain endothelial cells crossing and the cellular uptake by astrocytes were directly observed by TEM. MWNT- $\mathrm{NH}_{3}{ }^{+}$accumulation in endocytic vesicles and the reduced uptake following $4^{\circ} \mathrm{C}$ treatment together confirmed the involvement of energy-dependent cellular uptake. The transcytosis process was quantified by $\gamma$-scintigraphy using radiolabeled MWNT- $\mathrm{NH}_{3}{ }^{+}$in which maximum transport of $13 \%$ of MWNT- $\mathrm{NH}_{3}{ }^{+}$ was achieved after $72 \mathrm{~h}$ incubation.

"While stereotactic injection offers precise and direct access to the brain, its unfavorable invasive nature renders necessary the need for a systemic administration route to facilitate clinical translation of functionalized carbon nanotubes."

$f$-CNTs have contributed to therapeutic strategies against different neuropathological disorders in vivo including Alzheimer disease [13], glioblastoma [14,15] and stroke $[16,17]$. The first observation of $f$-CNTs in brain was reported by Yang et al. where shortened SWNTs were given orally to mice daily for 10 days [13]. SWNTs were taken up by absorptive cells and macrophages in the intestinal wall but were also detected in other organs such as liver, brain and heart. SWNTs were observed in lysosomes of neurons and neurites by TEM. Furthermore, such SWNTs were able to deliver acetylcholine, which was physically mixed with SWNTs, leading to improvement in the learning and memory capability of an experimentally induced Alzheimer disease mouse model.

Stereotactic delivery of $f$-CNTs into the brain is the most common technique used in the early studies involving $f$-CNTs. Zhao et al. utilized $f$-CNT to deliver an immunoadjuvant $\mathrm{CpG}$, leading to an antiglioma effect [14]. $f$-CNTs conjugated to $\mathrm{CpG}$ oligonucleotides were fluorescently labeled with $\mathrm{Cy} 5.5$ and were injected intratumorally (it.) into an intracranial GL261 glioma. It was found that $f$-CNT enhanced the uptake of CpG in GL261 tumor cells, as detected by FACS, resulting in elevated levels of proinflammatory cytokines and reduced tumor growth. Lee $e$ t al. reported that amine-modified SWNT ( $a$-SWNT) has the potential to protect neurons from injury using a rat stroke model [17]. Rats were given $a$-SWNTs via lateral ventricle injection before the induction of stroke. a-SWNTs-treated animals were found to have smaller infarct regions in the brain compared with the PBStreated group. $a$-SWNTs-treated animals performed better in the behavioral functions, assessed by RotaRod treadmill test, indicating the enhancement of recovery from stroke injury. The work conducted by us further demonstrated that $f$-CNTs can deliver therapeutic Caspase-3 siRNA into brain using a rat stroke model. $f$-CNTs-mediated delivery of siCas 3 via cortical injection successfully improved the antiapoptotic effect in the endothelin-1-induced stroke model, which was accompanied with promoted functional motor recovery. Internalization of $f$-CNTs by neuron cells were also observed by TEM at $48 \mathrm{~h}$ after injection.

Concerns have been raised whether there are adverse effects and inflammatory responses induced as a consequence of the intracranial injection itself and the introduction of $f$-CNTs into the brain. It is also important to understand the fate of $f$-CNTs after interaction with neuronal tissues, following stereotactic injection. We have investigated the in vivo biodegradation of $f$-CNTs by TEM [18], and also compared two types of $f$-CNTs in terms of their distribution patterns in relation to the possible inflammatory reactions [19] after stereotactic injection. TEM images revealed that $f$-CNTs were taken up by different neural cells (e.g., microglia, neurons and astrocytes) and the cellular internalization patterns differed between $f$-CNTs with different fictionalization. Structural deformation of $f$-CNTs inside cells was observed as early as $48 \mathrm{~h}$ postinjection and some tubes suffered severe decomposition with complete loss of cylindrical structural features. $f$-CNTs with different fictionalization strategies exhibited varied levels of proinflammatory cytokines but all at significantly low levels. The results together once again demonstrated the biocompatibility of $f$-CNTs which is a requirement of a delivery vector.

While stereotactic injection offers precise and direct access to the brain, its unfavorable invasive nature renders necessary the need for a systemic administration route to facilitate clinical translation of $f$-CNTs. Also, taking advantage of the various adsorptive-mediated or receptor-mediated transcytosis pathways existing in $\mathrm{BBB}$, researchers have attempted to develop $f$-CNTs tagged with a brain-targeting ligand to improve brain delivery after intravenous injection. Ren et al. developed dual-targeting $f$-CNTs that carry the brain targeting ligand, Angiopep-2 (ANG) as well as the anticancer drug doxorubicin (DOX) [15]. Oxidized MWNTs (O-MWNTs) were coated with polyethylene glycol (PEG) with ANG conjugated to its terminal. DOX was adsorbed MWNT sidewalls. Mice were intravenously injected with those $f$-CNTs, and an enhanced brain uptake of DOX-O-MWNTs-PEG-ANG was 
observed compared with non-ANG and DOX alone counterparts, measured by DOX fluorescence in isolated brains. DOX-O-MWNTs-PEG-ANG increased the median survival time the most. Direct and quantitative measurements of $f$-CNTs uptake in the brain or tumor was however not available in this study. A recent study from our group has examined quantitatively the in vivo biodistribution of $f$-CNTs and the brain uptake following intravenous injection using $\gamma$-scintigraphy [12]. Capillary depletion confirmed the presence of MWNT-NH${ }_{3}{ }^{+}$in brain capillaries and also parenchyma. The radiolabeled MWNT- $\mathrm{NH}_{3}{ }^{+}$under investigation exhibited intrinsic properties to cross $\mathrm{BBB}$ both in vitro and in vivo. The results thus pave the way for future applications of $f$-CNTs as nanocarriers for brain delivery.

Unlike the conventional delivery, other strategies involving the innate immune system have been explored to deliver $f$-CNTs to tumor [20,21]. PEGylated SWNTs were conjugated to an RGD ligand against integrin $\alpha_{v} \beta_{3}$ expressed on the surface of tumor blood vessels [20]. SWNT conjugates were fluorescently labeled with Cy5.5, allowing the detection of SWNTs in blood samples and tumor tissues using FACS and intravital fluorescence microscopy, respectively. Following intravenous injection, a certain subset of monocytes was identified to engulf high amount of SWNTs, and such selective SWNT-enriched monocytes were found to infiltrate into tumor interstitium. Another study was carried out by intratumoral injection of MWNT, tagged with a fluorescent probe $\mathrm{PKH} 26$, using the GL261 glioma model [21]. Most MWNTs (75\%) were phagocytosed by the tumor macrophages, microglia, and $30 \%$ microglia were found to be MWNT-positive at $24 \mathrm{~h}$ after injection. The presence of MWNTs in

\section{References}

1 Iijima $S$. Helical microtubules of graphitic carbon.

Nature 354(6348), 56-58 (1991).

2 De Volder MFL, Tawfick SH, Baughman RH, Hart AJ. Carbon nanotubes: present and future commercial applications. Science 339(6119), 535-539 (2013).

3 Monaco AM, Giugliano M. Carbon-based smart nanomaterials in biomedicine and neuroengineering. Beilstein J. Nanotechnol. 5, 1849-1863 (2014).

4 Bianco A, Kostarelos K, Partidos CD, Prato M. Biomedical applications of functionalised carbon nanotubes. Chem. Commun. (Camb.) 7(5), 571-577 (2005).

5 Kostarelos K, Bianco A, Prato M. Promises, facts and challenges for carbon nanotubes in imaging and therapeutics. Nat. Nanotechnol. 4(10), 627-633 (2009).

6 Liu Z, Tabakman S, Welsher K, Dai HJ. Carbon nanotubes in biology and medicine: in vitro and in vivo detection, imaging and drug delivery. Nano Res. 2(2), 85-120 (2009). glioma was confirmed by immunohistochemistry at 48 $\mathrm{h}$ postinjection. The above-mentioned immune-based delivery accounts for another approach to enhance tumor delivery of $f$-CNTs.

To summarize, CNTs are considered as one of the most promising nanomaterials as drug and gene delivery carriers and there are increasing numbers of studies documented on the use of functionalized CNTs for brain delivery. Results so far have shown that proper fictionalization is essential to yield biocompatible CNTs which are able to deliver either drugs or genes into brain through local or systemic injection. Future work should focus on better understanding of the interaction of $f$-CNTs with the BBBs and neural tissues, and the uptake mechanisms taking place. Such information is beneficial for the design and development of $f$-CNTs-based delivery system, offering efficient and specific targeting delivery into brain.

\section{Financial \& competing interests disclosure}

The authors would like to thank funding from Biotechnology and Biological Sciences Research Council (BB/J008656/1), Worldwide Cancer Research (12-1054) and Wellcome Trust (WT103913MF). The authors have no other relevant affiliations or financial involvement with any organization or entity with a financial interest in or financial conflict with the subject matter or materials discussed in the manuscript apart from those disclosed.

No writing assistance was utilized in the production of this manuscript.

\section{Open access}

This work is licensed under the Creative Commons Attribution 4.0 License. To view a copy of this license, visit http://creativecommons.org/licenses/by/4.0/

7 Pantarotto D, Singh R, Mccarthy D et al. Functionalized carbon nanotubes for plasmid DNA gene delivery. Angew. Chem. Int. Ed. Engl. 43(39), 5242-5246 (2004).

8 Al-Jamal KT, Nerl H, Muller KH et al. Cellular uptake mechanisms of functionalised multi-walled carbon nanotubes by $3 \mathrm{D}$ electron tomography imaging. Nanoscale 3(6), 2627-2635 (2011).

9 Kateb B, Van Handel M, Zhang L, Bronikowski MJ, Manohara H, Badie B. Internalization of mwents by microglia: possible application in immunotherapy of brain tumors. NeuroImage 37(Suppl. 1), S9-S17 (2007).

10 Abbott NJ. Blood-brain barrier structure and function and the challenges for CNS drug delivery. J. Inherit. Metab. Dis. 36(3), 437-449 (2013).

11 Shityakov S, Salvador E, Pastorin G, Förster C. Bloodbrain barrier transport studies, aggregation, and molecular dynamics simulation of multiwalled carbon nanotube functionalized with fluorescein isothiocyanate. Int. J. Nanomedicine 10, 1703-1713 (2015). 
12 Kafa H, Wang JT-W, Rubio N et al. The interaction of carbon nanotubes with an in vitro blood-brain barrier model and mouse brain in vivo. Biomaterials 53(0), 437-452 (2015).

13 Yang Z, Zhang Y, Yang Y et al. Pharmacological and toxicological target organelles and safe use of single-walled carbon nanotubes as drug carriers in treating Alzheimer disease. Nanomedicine 6(3), 427-441 (2010).

14 Zhao D, Alizadeh D, Zhang L et al. Carbon nanotubes enhance CpG uptake and potentiate antiglioma immunity. Clin. Cancer Res. 17(4), 771-782 (2011).

15 Ren J, Shen S, Wang D et al. The targeted delivery of anticancer drugs to brain glioma by PEGylated oxidized multi-walled carbon nanotubes modified with angiopep-2. Biomaterials 33(11), 3324-3333 (2012).

16 Al-Jamal KT, Gherardini L, Bardi G et al. Functional motor recovery from brain ischemic insult by carbon nanotubemediated sirna silencing. Proc. Natl Acad. Sci. USA 108(27), 10952-10957 (2011).
17 Lee HJ, Park J, Yoon OJ et al. Amine-modified single-walled carbon nanotubes protect neurons from injury in a rat stroke model. Nat. Nano 6(2), 121-125 (2011).

18 Nunes A, Bussy C, Gherardini L et al. In vivo degradation of functionalized carbon nanotubes after stereotactic administration in the brain cortex. Nanomedicine (Lond.) 7(10), 1485-1494 (2012).

19 Bardi G, Nunes A, Gherardini L et al. Functionalized carbon nanotubes in the brain: cellular internalization and neuroinflammatory responses. PLoS ONE 8(11), e80964 (2013).

20 Smith BR, Ghosneliver Eid B, Rallapalli $\mathrm{H}$ et al. Selective uptake of single-walled carbon nanotubes by circulating monocytes for enhanced tumour delivery. Nat. Nano 9(6), 481-487 (2014).

21 Vanhandel M, Alizadeh D, Zhang L et al. Selective uptake of multi-walled carbon nanotubes by tumor macrophages in a murine glioma model. J. Neuroimmunol. 208(1-2), 3-9 (2009). 